\title{
Organización comunitaria, aprovechamiento forestal y mujeres recolectoras, en El Punto, Oaxaca, México ${ }^{1}$
}

\section{Community Organization, Forest Harvesting, and Women Collectors, in El Punto, Oaxaca, México}

\author{
Sabina Carvente-Acteopan \\ Colegio de Postgraduados, Montecillo, México. \\ sabina.virgo@colpos.mx |https://orcid.org/0000-0003-0767-8562 \\ Luz María Pérez-Hernández \\ Colegio de Postgraduados, Montecillo, México. \\ luzmaph@colpos.mx |https://orcid.org/0000-0002-3285-8357 \\ María Antonia Pérez-Olvera \\ Colegio de Postgraduados, Montecillo, México. \\ molvera@colpos.mx |https://orcid.org/0000-0002-6408-8641 \\ Hermilio Navarro-Garza \\ Colegio de Postgraduados, Montecillo, México. \\ hermnava@colpos.mx |https://orcid.org/0000-0002-8599-3448 \\ María Flores-Cruz \\ Universidad Autónoma Metropolitana, Unidad Iztapalapa, México. \\ mafc@xanum.uam.mx | https://orcid.org/0000-0003-3013-8097
}

Recibido: 5 de diciembre de 2019. Aprobado: 23 de mayo de 2020

DOI: $10.25100 /$ lamanzanadeladiscordia.v15i1.8710

Artículo de investigación

¿Cómo citar este artículo? / How to quote this article?

Carvente, Sabina, Pérez, Luz María, Peréz María Antonia, Navarro, Hermilio, y Flores, María. (2020). Organización comunitaria, aprovechamiento forestal y mujeres recolectoras, en El Punto, Oaxaca, México. La Manzana de la Discordia, 15(1), 145-170. doi:

10.25100/lamanzanadeladiscordia.v15i1.8710

\footnotetext{
${ }^{1}$ Este artículo se deriva del trabajo de tesis de maestría, de la primera autora, en el Posgrado Socioeconomía, Estadística e Informática-Desarrollo Rural del Colegio de Postgraduados, Campus Montecillo.
} 


\title{
Resumen
}

El objetivo de esta investigación fue describir y analizar la organización comunitaria y la participación de las mujeres en el aprovechamiento forestal y de los RFNM en El Punto, Oaxaca. La información se obtuvo mediante recorridos en campo, entrevistas a informantes clave y a mujeres de las UMA, y una encuesta a 24 recolectoras/es. Se identificaron dos estructuras organizativas que aprovechan los RFNM: a) Dos Unidades de Manejo de la Vida Silvestre (UMA) y un aviso de aprovechamiento, integrados por mujeres recolectoras y b) Grupo de recolectoras/es que colectan RFNM en temporada navideña. Las acciones para el manejo de bromelias y RFNM son diferenciadas. Las prácticas de las recolectoras de las UMA son menos adversas. Se recomienda acciones complementarias de ambos grupos para la recolección, acondicionamiento y conservación de los RFNM. La comunidad combina procesos de autogestión con la normatividad externa para el aprovechamiento del bosque, sin embargo; limita la participación de las mujeres, solo al aprovechamiento de los RFNM. Las mujeres participan por obtener un ingreso, aprender nuevas cosas y contribuir con el cuidado del bosque. Las estructuras organizativas identificadas deben integrarse a la estructura organizacional comunitaria para fortalecer la certificación del bosque y el aprovechamiento sustentable del mismo.

Palabras clave: Gestión comunitaria; Prácticas de conservación; Procesos organizativos.

\begin{abstract}
This research was aimed to describe and analyze community organization and the participation of women in forest harvesting and RFNM, including bromeliads in El Punto, Santa Catarina Ixtepeji, Oaxaca. The information was obtained through field trips, interviews with key informants and women members of the Wildlife Management Units (WMU) and a survey to 24 gatherers from the community. Two ways of organization were identified: a) Two WMUs and a group women who collect all around the year, and b) A group of gatherers, that collect RFNM during Christmas season. Different actions were found between the organization for the management of bromeliads and RFNM. The practices carried out by the women of the UMA are less adverse. It is recommended to establish complementary actions for the collection, conditioning and conservation of bromeliads and RFNM by harvesting groups. The community combines self-management processes with external regulations in
\end{abstract}


decision-making for the management of forest resources; however, the participation of women it limits only for the use of RFNM. Women participate to get income, learn and contribute in the care of the forest. Identified organizational structures should integrate to communitarian organization to strength the forest certification and its sustainable use.

Keywords: Community management; conservation practices; organizational processes.

\section{Introducción}

México posee una enorme diversidad biológica, expresada en diversos ecosistemas y especies; por lo que se ubica entre los cinco países "megadiversos" más importantes (CONABIO, 2006). Sus bosques poseen una riqueza forestal maderable y no maderable muy destacada. De acuerdo con la FAO (1992), los recursos forestales no maderables (RFNM) son definidos como bienes de subsistencia para el consumo humano e industrial, los cuales son derivados de recursos y biomasas forestales renovables, e incluyen: alimentos, bebidas, forrajes, combustibles, medicinas, miel, laca y seda; y servicios para fines de conservación y recreación. Ticktin (2004) y Tapia-Tapia y Reyes-Chilpa (2008) mencionan que los RFNM han sido comercializados por muchos años, ya que brindan la posibilidad de aumentar los ingresos familiares y el empleo en zonas rurales, donde las mujeres son usuarias importantes de los recursos forestales. López (2008) señala que dichos recursos constituyen medios de vida, seguridad alimentaria, salud e ingreso a través de la recolección, a pesar de que existen dificultades en su aprovechamiento y uso sustentable (Arnold y Ruiz, 1996; Tapia-Tapia y Reyes-Chilpa, 2008). En el manejo de los RFNM, cobra importancia la participación de las mujeres, ya que son quienes mayormente dependen de los ingresos por el aprovechamiento de estos recursos y tienen menos acceso a ingresos de otro tipo (Mai, Mwangi, y Wan, 2012). Por su parte, Vázquez (2015) menciona que tener a mujeres en puestos de liderazgo y como participantes activas en procesos de planeación contribuye a su empoderamiento, permite que expresen sus intereses y necesidades y ayudan a que las reglas se cumplan.

En México, se han identificado 950 especies vegetales de las que se obtienen RFNM útiles, y únicamente 10\% de estas se comercializa (Tapia-Tapia y Reyes-Chilpa, 2008); por lo que existe un gran potencial de aprovechamiento para los poseedores de esos recursos. Uno de los RFNM que se recolectan son las bromelias, que pueden ser epífitas, terrestres o saxícolas cuyas inflorescencias de colores vistosos, las hacen atractivas como plantas de ornato (Flores- 
Palacios y Valencia-Díaz, 2007; Miranda et al., 2007; Mondragón, Ramírez, Flores y García, 2011), y que además pueden tener usos ceremoniales, (Haeckel, 2008), medicinales, alimentarios y para la obtención de fibras (Sandoval-Bucio, Flores-Cruz y Martínez-Bernal, 2004; Hornung-Leoni, 2011; Estrella-Parra, Flores-Cruz, Blancas-Flores, Koch y AlarcónAguilar, 2019). Las bromelias son muy importantes para el funcionamiento de ciertos ecosistemas, ya que contribuyen a la riqueza de las especies, ocupando un lugar primordial dentro del ciclo de nutrimentos y establecen un gran número de interacciones con otros organismos (Stuntz, Ziegler, Simon y Zotz, 2002). Adicionalmente, su aprovechamiento es realizado como una alternativa para mejorar los ingresos de personas en comunidades rurales. Para El Punto, Oaxaca, el heno (Tillandsia usneoides) es una bromelia de gran importancia para las integrantes de la Unidad de Manejo de la Vida Silvestre (UMA), especialmente en temporada "navideña" (Mondragón-Chaparro, Villa-Guzmán, Escobedo-Sarti y FrancoMéndez, 2006 y Carvente-Acteopan, Pérez-Olvera, Flores-Cruz, Navarro-Garza y FloresHernandez, 2017) siendo una especie muy abundante en dos de las cuatro zonas de recolección con las que cuenta la comunidad (Carvente et al., 2017).

Se estima que cerca de $80 \%$ de los bosques en México están en manos de comunidades con concesión de tierras de uso colectivo (Meave, Romero-Romero, Salas-Morales, PérezGarcía y Gallardo-Cruz, 2012); por consiguiente, la autogestión para el bien común adquiere gran relevancia (Ostrom, 2009), ya que la identificación de objetivos y acciones comunes, aglutinan, convocan y movilizan hacia el esfuerzo y la acción colectiva (Rojo, Martínez, Azpiroz y Jasso, 2008). Las comunidades llevan a cabo un manejo colectivo del bosque que les permite compartir usos, beneficios y responsabilidades de sus recursos (Pagdee, Kim y Daugherty, 2006) a través de esquemas de organización basados en: a) el patrimonio natural para la producción material y reproducción social, fundada en los derechos de propiedad colectiva del bosque; b) los arreglos institucionales para la gestión de los recursos de uso común; c) el sistema de usos y costumbres de los pueblos indígenas que ahí habitan y; d) formas de gobernanza basadas en una mayor participación ciudadana para la toma de decisiones (Gasca, 2014).

Sekher (2001) menciona que las organizaciones locales son las herramientas a través de las cuales la participación del grupo de usuarios en la gestión de los recursos toma forma, ya sea en la elaboración de políticas o en hacer cumplir las regulaciones (elaboración de 
normas, condiciones de acceso y distribución de beneficios). En su mayoría, estas organizaciones están constituidas por hombres. Si bien la organización comunitaria, por usos y costumbres, tiene aspectos positivos que favorecen el manejo sostenible de los recursos de uso común superiores a las capacidades de las instituciones federales, esto no asegura que los intereses de la mayoría sean tomados en cuenta, al excluir a las mujeres y no promover su participación (Rojas-Serrano, 2017).

En el estado de Oaxaca, 274 comunidades poseen bosques; su utilización representa la fuente de ingreso más importante para cerca del 50\% de ellas (Gasca, López, Palomino y Malthus, 2010). En dichos bosques se ha documentado la presencia de 168 (Flores-Cruz y Granados, 2011) a 172 especies de bromelias (Espejo-Serna, López-Ferrari, Martínez-Correa y Pulido-Esparza, 2007), que en conjunto con otros RFNM, son aprovechadas como plantas de ornato en temporada decembrina y se comercializan en los principales mercados de la capital del estado (Mondragón, 2008).

En la comunidad de El Punto se utilizan diversos RFNM, entre ellos las bromelias, que son aprovechados por las familias de ejidatarios con derechos vigentes, desde hace más de 100 años, mediante un modelo organizativo de autogestión local. En 2008, se crearon dos Unidades de Manejo para la Conservación de la vida Silvestre (UMA) bajo la NOM-059SEMARNAT-2010 (Diario Oficial de la Federación, 30 de diciembre de 2010) y un aviso de aprovechamiento, constituidas por mujeres, con vínculos familiares (esposas-hijas) de comuneros, con derecho de bosque vigentes, las cuales son iniciativas gubernamentales para la conservación y utilización de los recursos, cuyo fin es promover esquemas alternativos de producción, compatibles con el cuidado del ambiente, a través del uso racional, ordenado y planificado de los recursos naturales renovables, que frenen o reviertan los procesos de deterioro ambiental (Gallina-Tessaro, Hernández-Huerta, Delfín-Alonso y González-Gallina, 2009), y que se han incorporado a la organización local, generando opciones complementarias para el aprovechamiento de los RFNM.

Es así como el presente artículo tiene como objetivo describir y analizar las formas organizativas comunitarias y la participación de las mujeres en el aprovechamiento de las bromelias y otros RFNM, en la comunidad de El Punto, Santa Catarina Ixtepeji, Oaxaca, y analizar su funcionalidad como estructuras locales en la gestión de los recursos forestales, para identificar las implicaciones de su funcionamiento en la conservación de estas especies. 


\section{Área de estudio}

Santa Catarina Ixtepeji, Oaxaca se divide en cuatro agencias municipales: Yuvila, Tierra Colorada, Santa Catarina Ixtepeji y El Punto. Obtuvo la confirmación de bienes comunales por resolución presidencial en 1964, con una dotación de poco más de 21,392 hectáreas. El 80\% del territorio del municipio se encuentra cubierto por bosque y de este, el $71 \%$ se destina para actividades de aprovechamiento forestal de recursos maderables y no maderables. Cuenta con 2,532 habitantes, de los cuales 735 son comuneros.

La investigación se realizó en la comunidad de El Punto (Figura 1). Sus coordenadas geográficas son $96^{\circ} 35^{\prime} 2^{\prime}$ N y $17^{\circ} 13^{\prime} 18^{\prime}$ W y la altura media de $2304 \mathrm{msnm}$. Está integrada por 501 personas, $44 \%$ son hombres y 56\% mujeres; la población cuenta con un promedio de 6 años de escolaridad, 6\% habla zapoteco. La población económicamente activa es de 52\% (INEGI, 2010). Las principales actividades económicas son: la producción forestal maderable, aprovechamiento de productos forestales no maderables (PFNM), producción de cultivos básicos, ganadería, floricultura, extracción de leña y servicios ecoturísticos (Gasca et al., 2010). 
Fuente: elaboración personal con datos de INEGI (2005).

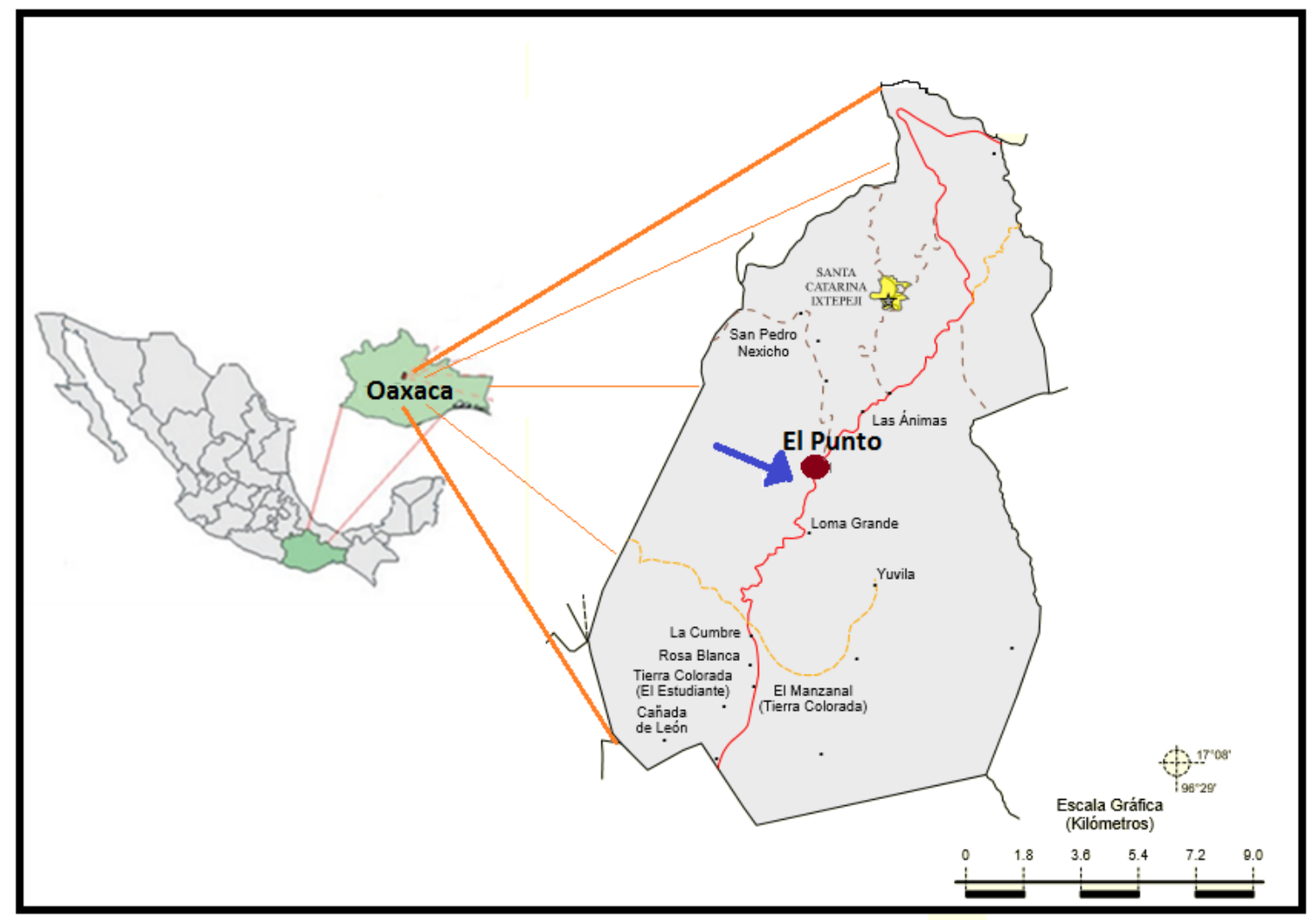

Figura 1. Localización de la comunidad de El Punto, Santa Catarina Ixtepeji, Oaxaca

\section{Estrategia metodológica}

El trabajo de campo se efectuó durante 2014 y 2015, periodo en el que se hicieron tres recorridos para identificar las zonas de recolección y observar el proceso de colecta de bromelias y otros RFNM. Se aplicó una encuesta piloto a seis comercializadores/as, en la Central de Abastos de Oaxaca y entrevistas a siete informantes clave (Comisariado de Santa Catarina Ixtepeji; cinco mujeres integrantes de las UMA y miembros del Consejo de Vigilancia), por ser los actores principales de la comunidad para la gestión de los recursos. Adicionalmente, se realizó una encuesta a 24 de las/os 27 recolectoras/es que ejercieron el permiso de recolección en 2014.

Para el análisis de los datos, se consideraron las siguientes variables de estudio: organización comunitaria, gestión, comercialización y conservación de las bromelias. La organización comunitaria consideró la estructura organizacional y las funciones de las figuras organizativas existentes; la gestión está relacionada con la definición de objetivos, los 
procesos administrativos y reglas de operación para la recolección y venta; la comercialización consideró organización para la venta, lugares de venta y precio; y la conservación consideró el análisis de la influencia de las prácticas realizadas por las dos formas organizativas en el aprovechamiento y conservación de las bromelias, considerando el grupo de mujeres y el grupo de recolectoras/es. La información obtenida en el trabajo de campo se procesó en una base de datos en Excel y el análisis de los resultados obtenidos se hizo mediante SPSS.

\section{Organización comunitaria para el aprovechamiento de los RFM y RFNM}

El municipio de Santa Catarina Ixtepeji se rige por el sistema de usos y costumbres, bajo régimen comunal. Para el manejo, aprovechamiento y conservación del bosque, cuentan con una estructura organizativa que refleja la amplia gama de actividades que se llevan a cabo. Por lo tanto la organización es un elemento estratégico en el desarrollo de la localidad como lo mencionan Pérez-Hernández, Figueroa-Sandoval, Díaz-Puente y Almeraya-Quintero. (2011).

De acuerdo con la información proporcionada por el Comisariado de bienes comunales, la comunidad cuenta con una estructura representativa autorizada por el Estatuto Comunal de Santa Catarina Ixtepeji, integrada por la Asamblea General de Comuneros, el Consejo de Administración constituido por el Comisariado de Bienes Comunales y el Consejo de Vigilancia y, a partir de 1992, un Consejo Consultivo que apoya las actividades de los órganos de representación (Figura 2). 
Fuente: elaboración personal con datos de investigación de campo.

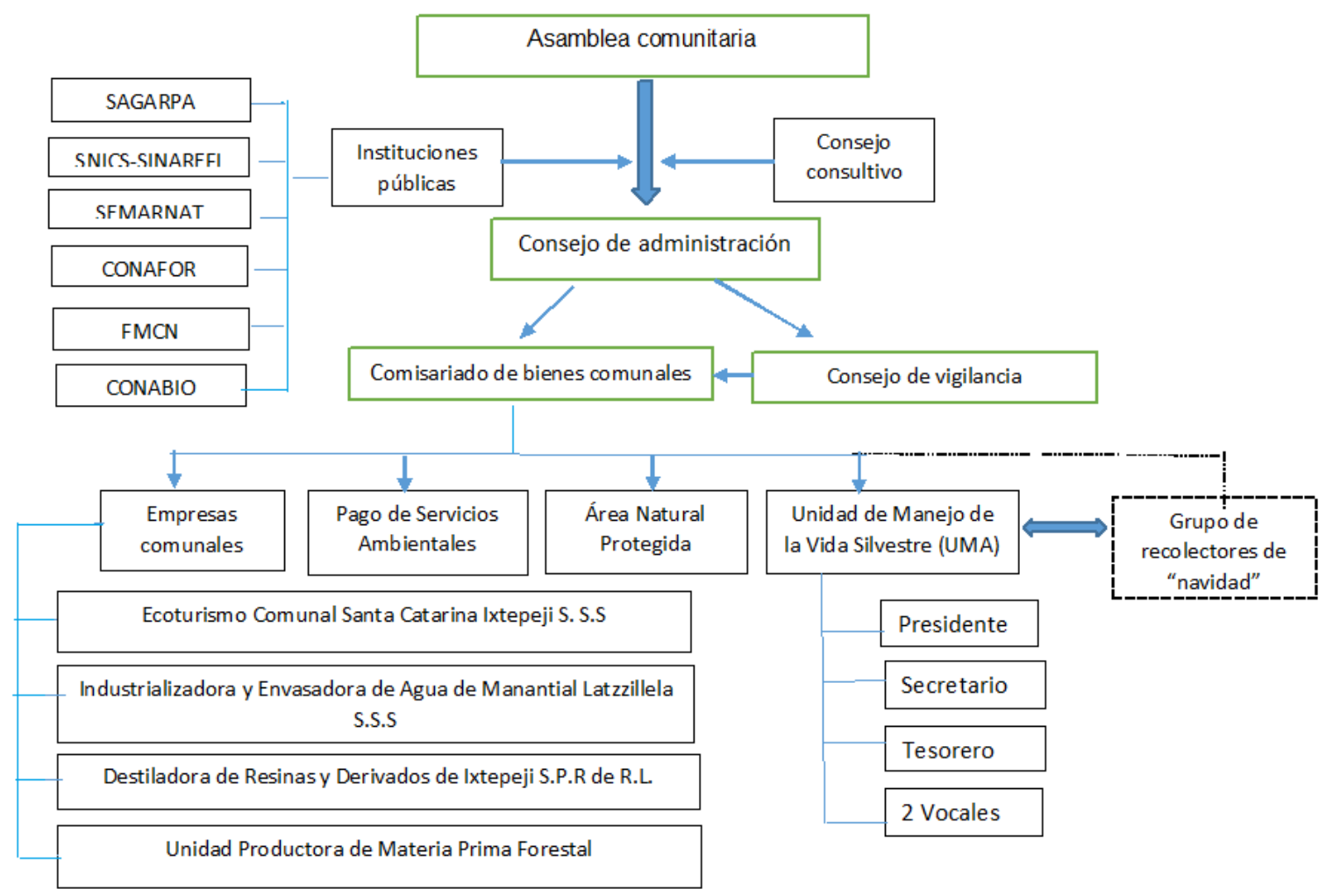

Figura 2. Estructura organizativa de la Comunidad Agraria de "Santa Catarina Ixtepeji"

Dependiendo de la estructura de representación, existen estructuras específicas operativas para el aprovechamiento y gestión de los recursos naturales (Figura 2). Tienen cuatro empresas comunales de aprovechamiento forestal, un área natural protegida, dos UMA y un aviso de aprovechamiento. Las UMA corresponden a bromelias y al hongo blanco (Tricholoma magnivelare Peck), y el aviso de aprovechamiento para las especies de bromelias que no se encuentran en la NOM-059 (Diario Oficial de la Federación, 30 de diciembre de 2010). Además, un grupo de comuneros para el aprovechamiento de RFNM. Adicionalmente, la comunidad recibe pago por servicios ambientales y financiamiento del Fondo Mundial de Conservación de la Naturaleza (FMCN). El responsable de coordinar las actividades de las diversas empresas comunales es el Comisariado de Bienes Comunales. Sin embargo, solo en tres de estas estructuras participan mujeres, las UMA, tanto la de bromelias, como la de hongos y en el grupo de recolectoras/es; en este último, los derechos para el aprovechamiento 
se ejercen por comunero (con derechos vigentes), y las mujeres que participan son esposas o familiares del comunero o viudas de comuneros y, por lo tanto, posesionarias de los derechos del esposo. Al respecto, Vázquez (2015) señala que para el caso de Hidalgo, México, los hombres son los que aprovechan y comercializan la madera, mientras que las mujeres se limitan a utilizar y aprovechar los recursos forestales no maderables (RFNM). Brown y Lassoie (2010), Djoudi y Brockhaus (2011) y Vázquez (2015) mencionan que pese a que las mujeres son usuarias muy importantes de los recursos forestales no maderables, su participación en la gobernanza forestal es limitada.

\section{Organización para aprovechamiento de bromelias}

En la comunidad se identificaron dos estructuras o formas organizativas para el aprovechamiento y conservación de bromelias y otros PFNM: .a) La organización formal para la extracción de bromelias es a través de las UMA “Catopsis” (EX-00011-OAX), autorizada en 2008, que permite la recolección de las especies Tillandsia carlos-hankii y Catopsis berteroniana y, el Aviso de Aprovechamiento "Bromelias" (INT-105-OAX) que autoriza la venta de todas las especies de bromelias de la localidad. En la práctica, funcionan como una sola estructura. El grupo está integrado por cinco mujeres de la comunidad, con edad promedio de 48 años y 6 años de escolaridad. El 60\% son casadas y el resto son viudas. Todas se dedican al hogar como principal actividad y al comercio, en segunda instancia, por lo cual las actividades que realizan en esta organización son complementarias. Las integrantes de las UMA funcionan mediante un Comité Directivo constituido por una presidenta, secretaria, tesorera y dos vocales, quienes establecen su plan de trabajo y toman las decisiones sobre el funcionamiento y actividades de las UMA. Los puestos son rotativos. El Comisariado de Bienes Comunales funge como representante legal, sin embargo, no tiene incidencia en la operación interna de las UMA. Su participación es para la gestión de apoyos y la presentación de los informes anuales de aprovechamiento, ante SEMARNAT y PROFEPA.

b) El grupo denominado "recolectoras/es de navidad" está formado por hombres y mujeres de la comunidad que recolectan bromelias y otros RFNM en la época decembrina (124 de diciembre). El grupo se constituye cada año por los comuneros con derechos vigentes, en el cual pueden participar el comunero o su esposa o su hijo (a). La participación de las mujeres (esposas o hijas) y jóvenes es siempre en representación de los comuneros. Al grupo se unen las mujeres de las UMA. El número y los integrantes de este grupo se modifican 
anualmente, y depende de la decisión de los comuneros y su familia de participar cada año. De las 24 personas recolectoras entrevistadas, el 83\% son mujeres. La edad promedio de las recolectoras fue 51 años $(\sigma=12,2)$ y de los recolectores 46 años $(\sigma=12)$; una escolaridad de 6 y 7 años; y un tiempo promedio de realizar la recolección de 31 y 30 años respectivamente. El $20 \%$ de las recolectoras no sabe leer ni escribir, mientras que todos los recolectores si lo hacen. El 100\% de las recolectoras se dedican al hogar como principal actividad, en tanto que el $50 \%$ de hombres se dedican a la agricultura y el otro $50 \%$ de ellos se dedican al comercio. La recolección de RFNM es una actividad complementaria. Este grupo no tiene una estructura formal, ni reglamento interno; su actuación se basa en un permiso anual que otorga el Comisariado de Bienes Comunales, en el cual se estipulan las especies y las cantidades de RFNM autorizadas para la recolección.

\section{Funcionamiento de las UMA}

Las integrantes de las UMA tienen un reglamento interno que regula el ingreso, las actividades de las integrantes y las sanciones. Esta forma organizativa funciona durante todo el año. Las integrantes realizan la recolecta de bromelias únicamente en cuatro zonas específicas: La Curva de San Miguel, Reynoso, La Cruz de Yovaneli y La Petenera; las dos primeras zonas se caracterizan por ser más diversas y abundantes para la recolección, las otras dos porque tienen especies de colores llamativos y especies exclusivas de esas zonas.

Las integrantes de las UMA acuden juntas al bosque a recolectar la(s) especie(s) que necesitan para mantener su inventario. Aproximadamente van cada 15 días y recolectan, de acuerdo con la normatividad que rige a las UMA, las especies permitidas en su plan de manejo y solamente aquellas que estén caídas de los árboles.

Para el manejo de las especies recolectadas, las mujeres de las UMA operan un vivero comunitario llamado "Las Bromelias" ubicado a pie de carretera, donde se acondicionan y comercializan las bromelias recolectadas y las sobrantes de la temporada navideña, incluyendo especies de otras zonas, aumentando así la diversidad existente en el vivero (20 especies y cerca de 1000 individuos, de manera permanente). Las bromelias se venden en macetas, troncos o ramas a un precio aproximado de $\$ 35$ durante todo el año, precio superior al manejado en época navideña, que en algunos casos llega a ser de \$5 También han participado en ferias nacionales, a las cuales han sido invitadas para la comercialización de las plantas. Dados los escasos ingresos generados por esta actividad, las mujeres integrantes de las UMA, 
se unen al grupo de recolectoras/es en temporada decembrina, por lo que llevan a cabo las mismas prácticas que realiza el grupo de "recolectoras/es de navidad", lo que se contrapone con su objetivo como organización. Al respecto, ellas han sugerido en asamblea comunitaria que las prácticas de manejo que ellas realizan durante el año, se apliquen por los "recolectora/es de navidad"; sin embargo, no han tenido una respuesta positiva.

\section{Reglas y normas para el aprovechamiento de RFNM por el grupo de "recolectoras/es de navidad"}

El aprovechamiento comunitario de los RFNM representa una alternativa para mejorar el ingreso de recolectoras/es, quienes indicaron que tienen derecho de extraer y comercializar bromelias como el paxtle blanco o heno (Tillandsia usneoides (L.) L.) y otros RFNM, entre ellos el paxtle amarillo (Squamidium sp. Broth), tapete (Polytrichum sp. Hedw) y espumitas (líquenes).

La cantidad de recolección es establecida mediante un permiso expedido por el Comisariado de Bienes Comunales, y avalado ante SEMARNAT bajo la NOM-011SEMARNAT-1996 (Diario Oficial de la Federación, 23 de abril del 2003), la cual establece criterios y especificaciones para el aprovechamiento, transporte y almacenamiento de musgo, heno y doradilla (Sellaginellla sp. P. Beauv).

El permiso se extiende a los comuneros con derechos vigentes, o a un familiar autorizado por él; no tiene costo y es válido para un solo periodo de recolección. La autorización incluye: las áreas de recolección, los cuidados al bosque y las especies que pueden o no ser aprovechadas. Su incumplimiento conlleva a la pérdida de derecho de monte, lo que significa que el comunero no podrá aprovechar ningún recurso del bosque durante tres años. Con respecto a la familia Bromeliaceae, se tiene restricción absoluta para la bromelia de cruz (Tillandsia gymnobotrya Baker) y para el heno, se establece una cantidad de extracción por temporada, que para 2014 fue de $95-109 \mathrm{~kg}$ por recolector/a.

Durante la recolección, se extraen diversas especies de bromelias (Tabla 1), algunas de las cuales están sujetas a protección especial como la "mechudita" (Tillandsia carlos-hankii Matuda) y Catopsis berteroniana (Schult y Schult f. Mez) o "jarrita" de acuerdo a la NOM059-SEMARNAT-2010 (Diario Oficial de la Federación, 30 de diciembre de 2010), las cuales son recolectadas por su abundancia en el bosque sin que exista registro de las cantidades extraídas, ni del posible impacto en el ecosistema. 
Tabla 1. Especies de bromelias epifitas recolectadas en la comunidad de El Punto, Oaxaca

\begin{tabular}{cll}
\hline $\begin{array}{c}\text { Recolectoras/es } \\
(\mathbf{\%})\end{array}$ & Especie recolectada & Nombre común \\
\hline 36 & Tillandsia carlos-hankii & "mechudita" \\
18 & Catopsis berteroniana & "jarrita" \\
12 & Tillandsia oaxacana & "florecita roja" \\
10 & Tillandsia magnusiana & "brujitas" \\
6 & Tillandsia plumosa & "magueyito" \\
12 & Otras especies & "magueyitos" \\
$100(95-109 \mathrm{~kg} /$ recolector/a) & Tillandsia usneoides & "heno" \\
\hline
\end{tabular}

Fuente: Elaboración personal, con datos de investigación de campo.

La recolección se realiza en las cuatro zonas donde se les tiene permitido el aprovechamiento a las integrantes de las UMA, de donde se extraen bromelias que están sobre los árboles en diferentes etapas fenológicas y cantidades variables. Recolectoras y recolectores acuden al bosque en familia, dos veces por semana con jornadas de $7 \mathrm{~h}$.

Las bromelias se transportan en costales o redes junto con otros RFNM. El consejo de vigilancia verifica las especies recolectadas y cobra una cuota (pasle amarillo \$25/costal, heno $\$ 23 /$ costal y tapete \$30/paca); además otorga una nota foliada reconocida por SEMARNAT. Recolectoras y recolectores acondicionan los RFNM para la venta en su hogar; el 8\% de ellas o ellos vende sus RFNM a otras u otros recolectoras/es y el 92\% los comercializa directamente en la central de abasto y en el mercado 20 de noviembre, en la ciudad de Oaxaca. Doce recolectoras/es pertenecen a La Unión de Expendedores de Pasle y Musgo de la Sierra, cuatro al Frente de Organizaciones Sociales y Comerciantes del Estado de Oaxaca y seis venden sus RFNM de forma ambulante con o sin permiso del municipio.

Las bromelias son consideradas como un recurso complementario. Se comercializan con un valor de 5 a 25 pesos por pieza, no representan el principal ingreso en la venta de RFNM, a excepción del heno que es considerado el tercer RFNM en importancia económica.

Una estrategia de las y los recolectoras/es para aumentar las ventas es diversificar los RFMN que ofrecen, por lo que ofertan otras especies de bromelias en etapa de floración, que adquieren en otras comunidades como perico (Tillandsia punctulata Schltdl y Cham), lechuga (Tillandsia multicaulis Steud) y florecita roja (Tillandsia oaxacana L.B. Sm.). Otras estrategias son: a) arreglos florales de bromelias combinadas con crasuláceas y musgo; b) troncos con bromelias en diferentes etapas de desarrollo; y c) arreglos en los pesebres con 
bromelias en floración. Una vez terminada la venta de temporada navideña, las bromelias que sobran se tiran, no se reincorporan al bosque, ni se llevan al vivero comunitario.

\section{La organización comunitaria y el aprovechamiento forestal}

La organización para el aprovechamiento comunal de los recursos forestales, hídricos y paisajísticos en Santa María Ixtepeji es compleja en virtud de la diversidad de actividades que se realizan en dicho municipio y con los que cuenta la comunidad, de donde se derivan diversas estructuras con fines específicos que están encaminadas al uso y protección de los recursos del bosque, no solo a través del aprovechamiento de la madera sino, como lo señala Gasca (2014), a través de actividades complementarias como el ecoturismo comunitario, el envasado de agua, la recolección de resina y la extracción de RFNM.

Uno de los RFNM que se aprovechan son las bromelias, como complemento de la extracción de otros recursos, sin que sean las de mayor importancia para recolectoras/es y para la comunidad, por lo que existen deficiencias en su manejo, derivadas del incumplimiento a normatividad y la falta de un plan de manejo para algunas especies en particular.

\section{Las Unidades de Manejo Ambiental}

Existe muy poca participación de la comunidad en esta organización; por un lado, el reglamento que rige las actividades de las integrantes es muy rígido, lo que desincentiva el interés por participar, pues no se ve como una actividad que vaya a retribuir un ingreso. En dicho reglamento se establece, entre otras cosas, que una o un nuevo integrante deberá realizar trabajo, sin remuneración durante un año, por lo que solamente se ha integrado una mujer desde su formación. Al respecto, Improven (2013) señala que las organizaciones deben reaccionar de forma rápida a las condiciones internas y externas en constante cambio, que obligan a flexibilizar las reglas y los procedimientos para una mejora continua; aspecto que debe ser considerado para que el grupo de mujeres de las UMA crezca. Tampoco hay interés de los hombres para participar en ella, ya que es vista como una actividad en la que se “entretienen" las mujeres; en este sentido, Zapata, Townsend, Rowlands, Alberti y Mercado (2002) señalan que las organizaciones de mujeres no solo deben superar la falta de experiencia en los procesos organizativos, sino también deben contrarrestar la ausencia de credibilidad que sus acciones merecen en la comunidad.

La autoridad comunal no percibe a las UMA como una fuente de ingreso para la comunidad; sin embargo, reconoce que es útil en el proceso de gestión por ser un requisito 
para obtener la certificación del bosque, lo que coincide con el caso de Uganda, donde la participación de las mujeres es vista como una formalidad para satisfacer a los donantes (Empaform, 2006). Por lo tanto, el trabajo de las mujeres se ve como una actividad que continúa en el área doméstica, sin darle la importancia que debe. Como lo señalan RojasSerrano et al. (2014), existen muy pocos espacios de acción y toma de decisiones referentes al bosque y son relegadas a tareas poco valoradas, consideradas de apoyo, lo que significa el reforzamiento de un sistema social, patriarcal, inequitativo e injusto.

Las integrantes de las UMA decidieron participar en ella porque vieron una posibilidad de ingreso, de aprender nuevas cosas y de conocer nuevos lugares. Zapata et al. (2002) y Merelas-Iglesias y Sánchez-Bello (2016) señalan que para las mujeres, la organización tiene múltiples significados, que van desde la solución de problemas básicos, así como poder avanzar culturalmente, dilucidar colectivamente problemas comunes, divertirse o descansar. Implica también el reconocimiento social y se incrementa la autoestima colectiva. Las mujeres consideran que su participación es una manera en la que ellas pueden colaborar en el cuidado del bosque, al recolectar y cuidar las plantas caídas de los árboles de manera natural en el vivero y no extraer plantas que se encuentran sobre los árboles. Aun cuando se espera que el establecimiento de una UMA, además de preservar la biodiversidad, genere oportunidades de diversificación económica para el sector rural (Weber, García-Marmolejo y Reyna-Hurtado, 2006), no ha sido este el caso, pues desde su creación, las mujeres no han tenido ganancias sustanciosas por esta actividad, pero independientemente del énfasis que las mujeres le dan a lo propiamente económico, para ellas los procesos de socialización y reconocimiento como mujeres rurales tienen valor por sí mismos (Mora, Fernández, y Ortega, 2016). En el ámbito productivo, representa un reto importante debido a las tensiones entre una racionalidad que se articula a partir de valores y códigos comunitarios y otra que se deriva de las relaciones mercantiles (Gasca, 2014).

La estructura administrativa de las UMA limita su autonomía, ya que la gestión de un proyecto o la solicitud de un apoyo la deben hacer a través del representante legal quien no está inmerso en las actividades de las UMA, no conoce sus necesidades, ni existe el interés para gestionar recursos para esta organización que no representa fuente de ingresos para los comuneros. Por otro lado, dada la normatividad que les aplica como comunidad agraria, no 
pueden formalizar otra organización independiente donde alguna de ellas sea la representante legal, por lo que se limitan sus opciones para poder impulsar la actividad.

El plan de manejo de las UMA no permite la propagación de las especies, por lo que su volumen de venta es muy limitado. Cuando han contactado con clientes potenciales, no pueden abastecer las cantidades requeridas y menos en un flujo constante. El precio al que se venden las bromelias en el vivero y en las ferias a las que asisten es superior al precio en los mercados; al respecto, Gómez, Mondragón y Méndez (2015) mencionan que el sobreprecio se debe a la "diferenciación", término que se refiere al apoyo para la conservación del bosque, del cual es partícipe el consumidor, aunque no es suficiente para pagar los gastos que se generan por el cuidado y comercialización de las plantas.

Aunque recibieron apoyo para la creación de las UMA y la elaboración del plan de manejo, nadie les da seguimiento, la capacitación que han recibido es esporádica y no cuentan con asesoría técnica o administrativa. En general, la problemática de esta organización es similar a la sugerida por Gallina et al. (2009) para otras UMA. Adicionalmente, Zapata et al. (2002), al referirse a organizaciones de mujeres, menciona que la falta de experiencia en el trabajo colectivo, nula representación en cargos decisorios, además de los problemas de género, familiares, sociales y comunitarios obstaculizan la acción de las mujeres. Por lo tanto, se requiere capacitación y asesoría técnica en aspectos de comercialización, gestión de recursos y organización que les permita manejarse de manera más eficiente; además de trabajo con ellas en temas de género.

Dados los escasos ingresos generados por esta actividad, las integrantes de las UMA se unen al grupo de recolectoras/es en temporada decembrina, lo que se contrapone a las prácticas que como organización realizan, y al sentido de conservación del bosque, ya que ni ellas mismas respetan los estatutos que las rigen; aun cuando es contradictorio su actuar, lo hacen para percibir un ingreso adicional.

La comunidad argumentó los usos y costumbres, y el derecho de propiedad sobre los recursos para su aprovechamiento, como la justificación para no realizar las prácticas sugeridas por las integrantes de las UMA. Al respecto, Gasca (2014) documenta que dentro de las comunidades se advierten procesos que muestran un deterioro de las relaciones comunitarias, en tanto que la praxis de la gobernanza resulta un proceso que tiende a 
redimensionarse en el marco de fenómenos de exclusión y de distribución desigual del poder y de los recursos generados.

\section{Grupo de "recolectoras/es de navidad"}

El grupo de "recolectoras/es de navidad" ven el aprovechamiento de RFNM como una alternativa para mejorar su ingreso en temporada navideña, pero como una actividad individual no como grupo, no tienen objetivos comunes que los aglutine para obtener beneficios compartidos, pues es una agrupación temporal que no está constituida legalmente y cuyos integrantes cambian año con año, lo que no permite mayor integración, ni siquiera la intención de trabajo en equipo.

Al igual que las integrantes de las UMA, la mayor parte de "recolectoras/es de navidad" son mujeres. Al respecto, la FAO (1992) y Schreckenberg y Marshall (2006) citan que las mujeres hacen mayor uso de los RFNM y ponen de manifiesto la importancia de las mujeres en la conservación de los recursos naturales (Rojas-Serrano et al., 2014).

El grupo de "recolectoras/es de navidad" se rige por la normatividad interna de la comunidad y el plan de manejo del bosque que tienen autorizado, donde se señalan las restricciones en cuanto al aprovechamiento de ciertas especies, lo que de acuerdo con Gasca et al. (2010), constituye un regulador externo de los esquemas de aprovechamiento local. Tal es el caso del heno, especie que es considerada importante económica y culturalmente, debido a su uso en los nacimientos navideños (Mondragón-Chaparro y Villa-Guzmán, 2008), que se comercializa a gran escala en todo el país, lo que pone en peligro sus poblaciones (CONABIO, 2009). Sin embargo, también existen especies que, aunque están sujetas a protección especial, son recolectadas en la comunidad por su aparente abundancia. En este sentido, MondragónChaparro, Méndez-García y Ramírez (2016) señalan que la recolección de bromelias silvestres puede llegar a tener un fuerte impacto en la conservación de las especies comercializadas debido a la pérdida de individuos adultos que son los que proporcionan semillas para la reproducción; además no existe registro de las cantidades extraídas, ni del posible impacto en el ecosistema, como lo señala López (2008). De hecho, aun cuando se están incumpliendo las normas que las protegen, no existe ningún tipo de sanción por parte de las autoridades locales ni de las instancias gubernamentales encargadas de dicha regulación.

El grupo de recolectoras/es también extraen otras especies de bromelias en diferentes etapas fenológicas en cantidades variables que no tienen restricciones para su 
aprovechamiento. En este sentido, la falta de regulación puede poner en peligro sus poblaciones, por lo que se debe considerar efectuar estudios que contribuyan a su conservación. Ticktin (2004) indica que para proteger los RFNM de la extracción se requiere 1) la identificación de los regímenes de cosecha óptimos; 2) una estimación precisa de los límites máximos de recolección; y 3) la implementación de estos límites por recolectoras/es locales.

La venta de bromelias es una actividad que se realiza de manera individual y no hay ninguna forma de organización dentro de la comunidad para realizarla; sin embargo, una parte importante de recolectoras/es ha tenido que integrarse a alguna organización de comerciantes en la ciudad de Oaxaca para poder vender sus productos en los mercados locales, ya que de no hacerlo, no tendrían acceso a estos.

Miranda et al. (2007) indican que se manejan precios muy bajos para este recurso, ya que el tiempo de crecimiento estimado para que una bromelia llegue a floración y sea posible su comercialización es de 9 a 16 años (Flores-Palacios y Valencia-Díaz, 2007; Toledo-Aceves, García-Franco y López-Barrera, 2014). Por otro lado, no hay estudios que permitan definir el costo de extracción y el precio de venta de una bromelia, por lo que se comercializa al precio que impone el mercado, provocando la desvalorización de este tipo de recursos, al no considerar otros elementos. En términos de Aguilera (2006), el valor más importante de la naturaleza es el de su propia existencia y el de las funciones que cumple en el mantenimiento de la vida, en los procesos de producción y en la conformación de la cultura.

Para el grupo de recolectoras/es, el ingreso por la venta de bromelias no es significativo ni el de mayor importancia, además de que no perciben que puede haber un daño al bosque por su extracción dada su abundancia, aunque algunas de las especies se encuentren bajo protección. Es por ello que prácticamente no se realiza ninguna actividad de conservación de este recurso, tampoco existen estudios que permitan identificar el daño causado por el aprovechamiento actual, ni se toman las medidas necesarias por parte de la comunidad para regular la extracción de las bromelias. 


\section{Reflexiones finales}

El aprovechamiento de bromelias y de otros RFNM en la comunidad de El Punto, representa una fuente de ingreso complementario tanto para las mujeres que integran las UMA y el aviso de aprovechamiento durante todo el año, así como para el grupo de "recolectoras/es de navidad" integrado por $56 \%$ de mujeres y $44 \%$ de hombres.

A las mujeres recolectoras integrantes de las UMA, el trabajo en equipo para el aprovechamiento de los RFNM incluyendo las bromelias, les ha permitido avanzar colectivamente mediante la participación en capacitaciones para el reconocimiento, manejo y acondicionamiento de las bromelias, manejo de inventario, elementos administrativos, entre otros. Además, les ha permitido participar en ferias y eventos científicos como vendedoras invitadas, lo cual les ha permitido el desarrollo de habilidades de comunicación, toma de decisión, aspectos de logística, mercadeo, entre otras, fomentando con esto el reconocimiento social del trabajo que realizan. Acciones que les ha permitido empoderarse y reconocerse como actoras importantes en el manejo de los RFNM y en el cuidado del bosque.

Aun cuando la participación de las mujeres en las UMA y en el aviso de aprovechamiento les ha generado beneficios organizativos y sociales, el aprovechamiento de los RFNM no representa una fuente de ingreso suficiente para cubrir sus necesidades, lo que las obliga a unirse al "grupo de recolectoras/es en época decembrina," para quienes no existe restricción sobre la recolección de las especies, pese a que hay señalamiento de uso limitado para algunas especies; haciendo a un lado las prácticas de conservación que realizan durante todo del año. De esta forma, las estructuras organizativas de aprovechamiento de RFNM presentan retos en las prácticas de recolección y conservación de las especies.

Las prácticas que llevan a cabo las mujeres de las UMA son menos adversas que las que realizan el grupo de recolectoras/es, por lo que se recomienda establecer acciones complementarias de recolección, acondicionamiento y conservación de las bromelias y los RFNM por los grupos de aprovechamiento. Además es necesario realizar acciones dentro de la comunidad que promuevan la inclusión de las mujeres en la gobernanza forestal y le den mayor importancia a estas estructuras organizativas a nivel de comunidad, ya que son estructuras necesarias y estratégicas para la certificación del bosque y para el manejo sustentable de los recursos forestales. 


\section{Referencias bibliográficas}

Aguilera, Uclés. (2006). El valor económico del medio ambiente. Revista Ecosistemas, 15(2): 66-71.

Recuperado

de

https://www.revistaecosistemas.net/index.php/ecosistemas/article/view/187

Arnold, Michael, y Ruiz, Manuel. (1996). Framing the Issues Relating to Non-Timber Forest Products Research. En Manuel Ruiz y Michael Arnold (Editors), Current Issues in Non Timber Forest products Research, (pp. 1-18). Indonesia: Center for International Forestry Research. Recuperado de http://www.cifor.org/ntfpcd/pdf/ntfp-current.pdf

Brown, Caroline, y Lassoie, James. (2010). The Interaction Between Market Forces and Management Systems: A Case Study of Non-Wood Forest Products in the Humid Forest Zone of Cameroon. International Forestry Review, 12(1), 13-26. https://doi.org/10.1505/ifor.12.1.13

Carvente-Acteopan, Sabina, Pérez-Olvera, Antonia, Flores-Cruz, María, Navarro-Garza, Hermilio, y Flores-Hernandez, Noé. (2017). Diversidad y abundancia de bromelias epifitas en "El Punto" Santa Catarina Ixtepeji, Oaxaca. Revista mexicana de ciencias agrícolas, 8(especial18), 3.661-3.671. https://dx.doi.org/10.29312/remexca.v8i18.211

Traditional Management Units, The Base of Community Conservation in Morelos, Mexico. Revista Chapingo. Serie Ciencias Forestales y del Ambiente, 22(1), 7-27. https://doi.org/10.5154/r.rchscfa.2014.10.045

Comisión Nacional para el Conocimiento y Uso de la Biodiversidad (CONABIO). (2006). Capital natural y bienestar social. México: Comisión Nacional para el Conocimiento y $\begin{array}{lllll}\text { Uso de la } & \text { Biodiversidad. }\end{array}$ http://www.conabio.gob.mx/2ep/images/3/37/capital_natural_2EP.pdf

Comisión Nacional para el Conocimiento y Uso de la Biodiversidad (CONABIO). (2009). Bromeliaceae Tillandsia usneoides (L.) L. Heno. Recuperado de http://www.conabio.gob.mx/malezasdemexico/bromeliaceae/tillandsiausneoides/fichas/ficha.htm\#6

Diario Oficial de la Federación. (23 de abril del 2003). Norma Oficial Mexicana NOM-011SEMARNAT-1996 que establece los procedimientos, criterios y especificaciones para realizar el aprovechamiento, transporte y almacenamiento de musgo, heno y doradilla. Recuperado de http://www.profepa.gob.mx/innovaportal/file/3318/1/nom-011-semarnat- 
1996.pdf

Diario Oficial de la Federación. (30 de diciembre de 2010). NORMA Oficial Mexicana NOM059-SEMARNAT-2010, Protección ambiental-Especies nativas de México de flora y fauna silvestres-Categorías de riesgo y especificaciones para su inclusión, exclusión o cambio-Lista de especies en riesgo. Recuperado de http://www.profepa.gob.mx/innovaportal/file/435/1/NOM_059_SEMARNAT_2010.pdf

Djoudi, Houria, y Brockhaus, Maria. (2011). Is Adaptation to Climate Change Gender Neutral? Lessons from Communities Dependent on Livestock and Forests in Northern $\begin{array}{llll}\text { Mali. International } & \text { Forestry } & \text { Review, } & \text { 13(2), }\end{array}$ https://doi.org/10.1505/146554811797406606

Empaform. (2006). Participatory Forest Management Initiatives in Uganda: Key Implementation Concerns and Recommendations For Policy Actions. Empaform Policy Briefing Paper No. 1. Empaform, Kampala, Uganda.

Espejo-Serna, Adolfo, López-Ferrari, Ana, Martínez-Correa, Nancy, y Pulido-Esparza, Valeria. (2007). Bromeliad Flora of Oaxaca, México: Richness and Distribution. Acta Botánica Mexicana, 71-147. $\quad$ Recuperado de https://www.redalyc.org/pdf/574/57408105.pdf

Estrella-Parra, Edgar, Flores-Cruz, María, Blancas-Flores, Gerardo, Koch, Stephen, y Alarcón-Aguilar, Francisco. (2019). The Tillandsia Genus: History, Uses, Chemistry, and Biological Activity. Boletín Latinomericano y del Caribe de plantas Medicinales y Aromáticas, 18(3): 239-264.

Flores-Cruz, María, y Granados, Carolina. (2011). Bromeliaceae. En Abisaí García-Mendoza y Jorge Meave (Eds.), Diversidad florística de Oaxaca: de musgos a angiospermas (colecciones y lista de especies) (pp. 310-314). México: Instituto de Biología UNAM y CONABIO.

Flores-Palacios, Alejandro, y Valencia-Díaz, Susana. (2007). Local Ilegal Trade Reveals Unknown Diversity and Involves a High Species Richness of Wild Vascular Epiphytes. Biological conservation, (136), 372-387. https://doi.org/10.1016/j.biocon.2006.12.017

Gallina-Tessaro, Sonia, Hernández-Huerta, Arturo, Delfín-Alfonso, Christian, y GonzálezGallina, Alberto. (2009). Unidades para la conservación, manejo y aprovechamiento sustentable de la vida silvestre en México (UMA). Retos para su correcto 
funcionamiento. Investigación ambiental, 1(2), 143-152. Recuperado de http://www2.inecc.gob.mx/publicaciones/gacetas/627/unidades.pdf

Gasca, José. (2014). Gobernanza y gestión comunitaria de recursos naturales en la Sierra Norte de Oxaca. Región y Sociedad, 26(60), 89-120. Recuperado de http://www.scielo.org.mx/scielo.php?pid=S1870-9252014000300004\&script=sci_arttext

Gasca, José, López, Gustavo, Palomino, Bertha, y Malthus, Martín. (2010). La gestión comunitaria de recursos naturales y ecoturísticos en la Sierra Norte de Oaxaca. México: UNAM.

Gómez, Laura, Mondragón, Demetria, y Méndez, Elia. (2015). Aprovechamiento sustentable de Bromelias Epífitas: Propuesta comercial del vivero comunitario "Las Bromelias", Santa Catarina Ixtepeji. Oaxaca. Revista de Investigación académica sin frontera, 8(21), 1-20. Recuperado de http://revistainvestigacionacademicasinfrontera.com

Haeckel, Ingrid. (2008). The "Arco Floral”: Ethnobotany of Tillandsia and Dasylirion Spp. in a Mexican Religious Adornment. Economic Botany, 62(1), 90-95. Recuperado de http://www.jstor.org/stable/40390592

Hornung-Leoni, Claudia. (2011). Avances sobre usos etnobotánicos de las Bromeliaceae en Latinoamérica. Boletín Latinoamericano y del Caribe de Plantas Medicinales y Aromaticas, $\quad$ 10(4), 297-314. Recuperado de https://www.redalyc.org/pdf/856/85619300003.pdf

Improven. (2013). Organizaciones flexibles, la clave para tener éxito. Newsletter Allied Consultants. Europe-Improven Recuperado de https://www.improven.com/blog/organizativa-organizaciones-flexibles-la-clave-paratener-exito/

Instituto Nacional de Estadística y Geografía (INEGI). (2005). Prontuario de información geográfica municipal de los Estados Unidos Mexicanos Santa Catarina Ixtepeji, Oaxaca. Marco geoestadístico municipal 2005, versión 3.1. Recuperado de http://www3.inegi.org.mx/contenidos/app/mexicocifras/datos_geograficos/20/20363.pdf Instituto Nacional de Estadística y Geografía (INEGI). (2010). Datos geográficos de Oaxaca. Recuperado de http://www3.inegi.org.mx/sistemas/mexicocifras/datosgeograficos/20/20363.pdf

López, René. (2008). Productos Forestales No Maderables: importancia e impacto de su 
aprovechamiento. Revista Colombia Forestal, 11(1), 215-231. Recuperado de http://www.scielo.org.co/pdf/cofo/v11n1/v11n1a14

Mai, Yen Hoang, Mwangi, Esther, y Wan, Melinda. (2012). Gender Analysis in Forestry Research. Looking Back and Thinking Ahead. Center for International Forestry Research, (51), 1-6. Recuperado de http://www.jstor.org/stable/resrep01910

Meave, Jorge, Romero-Romero, Marco, Salas-Morales, Silvia, Pérez-García, Eduardo, y Gallardo-Cruz, Alberto. (2012). Diversidad, amenazas oportunidades para la conservación del bosque tropical caducifolio en el estado de Oaxaca, México. Ecosistemas, 21(1-2), 85-100. Recuperado de http://www.revistaecosistemas.net/index.php/ecosistemas/article/viewFile/29/25

Merelas-Iglesias, Tania, y Sánchez-Bello, Ana. (2018). El papel del asociacionismo de mujeres en el medio rural coruñés y los beneficios de la participación. Estudios Rurales, 8(16), 176-188. Recuperado de http://ppct.caicyt.gov.ar/index.php/estudiosrurales/article/view/13918/45454575759302

Miranda, Martha, Arellano, Juan, Salazar, Blanca, Hernández, Floriana, Quero, Raday, y Pérez, Lilia. (2007). Bromelias Ornamentales. Bases para el manejo comunitario de bromelias ornamentales. México: Grupo Autónomo para la Investigación Ambiental A. C.

Mondragón-Chaparro, Demetria. (2008). La comercialización navideña de bromelias epífitas en la ciudad de Oaxaca, México. Etnobiología, 6(1), 25-28. Recuperado de http://www.scielo.org.mx/-pdf/polib/n26/n26a10.pdf

Mondragón-Chaparro, Demetria, Méndez-García, Elia, y Ramírez, Ivon. (2016). Prioritizing the Conservation of Epiphytic Bromeliads Using Ethobotanical Information from a Traditional Mexican Market. Economic Botany, 70(1), 29-36. Recuperado de https://www.researchgate.net/publication/296686672

Mondragón-Chaparro, Demetria, Ramírez, Ivon, Flores, María, y García, José. (2011). La familia bromeliaceae en México. México: Universidad Autónoma Chapingo.

Mondragón-Chaparro, Demetria., y Villa-Guzmán, Dulce. (2008). Estudio Etnobotánico de las Bromelias epifitas en la comunidad de Santa Catarina Ixtepeji, Oaxaca, México. Poli Botánica, 26(1), 175-191. Recuperado de http://www.redalyc.org/-pdf/621/62102610.pdf Mondragón-Chaparro, Demetria., Villa-Guzmán, Dulce., Escobedo-Sarti, Guadalupe., y 
Franco-Méndez, Alma. (2006). La riqueza de bromelias epífitas a lo largo de un gradiente altitudinal en Santa Catarina Ixtepeji, Oaxaca, México. Naturaleza y Desarrollo, 4(2), 1316.

Mora, Gloria, Fernández, María, y Ortega, Sofia. (2016). Economic and Productive Associations and Empowerment of Women in Rural Areas: Multi-Active Mothers, Productive Partners and Country Women. Cultura-hombre-sociedad, 26(1), 133-160. https://dx.doi.org/10.7770/CUHSO-V23N1-ART1055

Organización de las Naciones Unidas para la Alimentación y la Agricultura (FAO). (1992). Productos forestales no madereros; posibilidades futuras. Roma, Italia: FAO. Recuperado de http://www.fao.org/docrep/019/t0431s/t0431s.pdf

Ostrom, Elinor. (2009). El gobierno de los bienes comunes: La evolución de las instituciones de acción colectiva. México: UNAM, Fondo de Cultura Económica.

Pagdee, Adcharaporn, Kim, Yeon-Su, y Daugherty, P. J. (2006). What Makes Community Forest Management Successful: A Meta-Study from Community Forests Throughout the World. Society and Natural Resources, 19(1), 33-52. Recuperado de http://www.tandfonline.com/doi/abs/10.1080/08941920500323260

Pérez-Hernández, Luz, Figueroa-Sandoval, Benjamín, Díaz-Puente, José, y AlmerayaQuintero, Silvia. (2011). Influencia de organizaciones en el desarrollo rural: caso de Salinas, San Luis Potosí. Revista Mexicana de Ciencias Agrícolas, 2(4), 515-527. Recuperado de https://www.scielo.org.mx/pdf/remexca/v2n4/v2n4a4.pdf

Rojas-Serrano, Coral. (2017). Transformaciones y continuidades en el manejo del bosque y relaciones en Santa Catarina Lachatao, Oaxaca. Universidad Nacional Autónoma de México, Centro Regional de Investigaciones Multidisciplinarias. 1ra Edición. México: Cuernavaca, Morelos.

Rojas-Serrano, Coral, y Martínez-Corona, Beatriz. (2017). Transformaciones y continuidades en el manejo y concepción del bosque y las relaciones de género en Santa Catarina Lachatao, Oaxaca. Agricultura, sociedad y desarrollo, 14(3), 451-478. https://doi.org/10.22231/asyd.v14i3.645

Rojas-Serrano, Coral, Martínez-Corona, Beatriz, Vázquez-García, Verónica, CastañedaSalgado, Patricia, Zapata-Martelo, Emma, y Sámano-Rentería, Miguel. (2014). Estrategias de reproducción campesina, género y valoración del bosque en Lachatao, 
Oaxaca México. Agricultura sociedad y desarrollo, 11(1), 71-92. https://doi.org/10.22231/asyd.v11i1.54

Rojo, Gustavo, Martínez, Rosa, Azpíroz, Hilda, y Jasso, Jesús. (2008). Desarrollo forestal comunal en las comunidades indígenas de México. En Rosa Martínez, Gustavo Rojo, Hilda Azpíroz, Eugenia Barba y Benito Ramírez (Eds.), Avances de investigación forestal y desarrollo sustentable, (pp. 25-52). México: Universidad Autónoma Indígena de México.

Sandoval-Bucio, Evgueny, Flores-Cruz, María, y Martínez-Bernal, Angélica. (2004). Bromelias útiles de México. Cactáceas y Suculentas Mexicanas, (49), 100-115.

Schreckenberg, Kathrin, y Marshall, Elaine. (2006). Los PFNM y las mujeres ¿Mejora en el ingreso y la posición social? En Elaine Marshall, Kathrin Schreckenberg y A.C. Newton (Eds.), Comercialización de Productos Forestales No Maderables. Factores que Influyen en el Éxito. Conclusiones del estudio de México y Bolivia e implicancias políticas para los tomadores de decisión, (pp. 83-86). Reino Unido: Centro Mundial de Vigilancia de la Conservación del PNUMA (UNEP-WCMC).

Sekher, Madhushree. (2001). Organized Participatory Resource Management: Insights from Community Forestry Practices in India. Forest Policy and Economics, 3(3), 137-154. Recuperado de http://www.sciencedirect.com/science/article/pii/S1389934101000600

Stuntz, Sabine., Ziegler, Christian., Simon, Ulrich., y Zotz, Gerhard. (2002). Diversity and Structure of the Arthropod Fauna Within Three Canopy Epiphyte Species in Central Panama. Journal of Tropical Ecology, 18(2), 161-176.

Tapia-Tapia, Estrella., y Reyes-Chilpa, Ricardo. (2008). Productos forestales no maderables en México: aspectos económicos para el desarrollo sustentable. Maderas y Bosques, 14(3), 95-112. Recuperado de http://www.redalyc.org/pdf/-617/61712189005.pdf

Ticktin, Tamara. (2004). The Ecological Implications of Harvesting Non-Timber Forest Products. Journal of Applied Ecology, 41(1), 11-21. https://doi.org/10.1111/j.13652664.2004.00859.x

Toledo-Aceves, Tarin, García-Franco, José, y López-Barrera, Fabiola. (2014). Bromeliad Rain: An Opportunity for Cloud Forest Management. Forest Ecology and Management, 329, 129-136. https://doi.org/10.1016/j.foreco.2014.06.022

Vázquez, Verónica. (2015). Manejo forestal comunitario, gobernanza y género en Hidalgo, 
México. Revista mexicana de sociología, 77(4), 611-635. Recuperado de https://www.redalyc.org/articulo.oa?id=32141548004

Weber, Manuel, García-Marmolejo, Gabriela, y Reyna-Hurtado, Rafael. (2006). The Tragedy of the Commons: Wildlife Management Units in Southeastern Mexico. Wildlife Society Bulletin, $\quad 34(5), \quad$ 1.480-1.488. https://doi.org/10.2193/00917648(2006)34[1480:TTOTCW]2.0.CO;2

Zapata, Emma, Townsend, Janet, Rowlands, Jo, Alberti, Pilar, y Mercado, Martha. (2002). Las mujeres y el poder contra el patriarcado y la pobreza. México: Colegio de Postgraduados y Plaza y Valdés. S. A. de C. V. 